\title{
Depinning Transition of a Two Dimensional Vortex Lattice in a Commensurate Periodic Potential
}

\author{
Violeta Gotcheva and S. Teitel \\ Department of Physics and Astronomy, University of Rochester, Rochester, NY 14627
}

(November 19, 2018)

\begin{abstract}
We use Monte Carlo simulations of the 2D one component Coulomb gas on a triangular lattice, to study the depinning transition of a $2 \mathrm{D}$ vortex lattice in a commensurate periodic potential. A detailed finite size scaling analysis indicates this transition to be first order. No significant changes in behavior were found as vortex density was varied over a wide range.
\end{abstract}

74.60.Ge, 64.60-i, 74.76-w

The theory of defect mediated melting of a two dimensional (2D) solid, introduced by Kosterlitz and Thouless (KT) [1] and developed by Nelson and Halperin (NH) [2] and Young 3 in the late 70's, has remained a topic of active investigation. Numerous numerical studies have reported conflicting results as to whether the $2 \mathrm{D}$ melting transition is indeed a 2nd order KT type transition, or whether it is 1st order [4. The most recent results have supported the KT scenario [5]. In the same paper [2] in which they developed this theory of $2 \mathrm{D}$ melting in a continuum, Nelson and Halperin also considered the case of a $2 \mathrm{D}$ solid in a commensurate periodic potential. In this case, they argued that the $2 \mathrm{D}$ solid would have two distinct phases: a "pinned" solid with long range translational correlations at low temperatures, and a "floating" solid with algebraic translational correlations, similar to that found in the continuum, at intermediate temperatures. By mapping the problem to a vector Coulomb gas, they argued that this depinning transition was a 2 nd order KT type transition similar to that of melting, with a universal discontinuous jump in the exponent $\eta$ characterizing the algebraic correlations of the floating solid phase.

Despite the wide attention given to the continuum 2D melting problem, this depinning transition has been very little studied. Only recently has the floating solid phase been observed in numerical studies of $2 \mathrm{D}$ vortex lattices [6], and related XY models [7]. In this paper we present the first detailed finite size scaling analysis of this depinning transition. We treat the specific case of logarithmically interacting $2 \mathrm{D}$ vortices. This is not only a system of considerable recent interest in connection with high temperature superconductors, but also has a unique advantage for numerical simulations: it is an incompressible system. We can simulate at constant density, yet there will still be a sharp transition temperature, rather than the finite temperature interval of coexisting phases that one would have for other interactions (should the transition be 1st order). We can therefore avoid the controversy, that arose in $2 \mathrm{D}$ melting simulations, as to whether one should use a constant pressure rather than a constant volume ensemble. For this vortex system, our results are quantitatively consistent with a 1st order depinning transition.

Our model is the one component classical Coulomb gas on a triangular lattice, given by the Hamiltonian,

$$
\mathcal{H}=\frac{1}{2} \sum_{i, j}\left(n_{i}-f\right) G_{i j}\left(n_{j}-f\right) .
$$

Here $n_{i}=0,1$ is the integer charge at site $i$ of an $L \times L$ periodic triangular lattice; $-f$ is a uniform background charge density; charge neutrality fixes the number of integer charges to $\sum_{i} n_{i}=f L^{2} . G_{i j}$ is the $2 \mathrm{D}$ Coulomb potential for a discrete triangular lattice with periodic boundary conditions [6], and the sum is over all pairs of sites. $G_{i j} \sim \ln \left|\mathbf{r}_{i}-\mathbf{r}_{j}\right|$ for distances large compared to the lattice spacing, but small compared to $L$. The charges $n_{i}$ model logarithmically interacting vortices in the phase of a superconducting wavefunction, $f$ is the number of applied magnetic flux quanta per unit cell of the triangular lattice, and restricting the vortices to the sites of the triangular lattice models the periodic pinning potential [6].

We study the Hamiltonian (1) using Monte Carlo (MC) simulations. Details of our simulation methods follow those described in Ref. [6]. Our main results are for the fixed vortex density of $f=1 / 100$.

To decide the order of the depinning transition at $T_{c}$, we study the finite size scaling of the energy density histogram $P(E)$ measured close to $T_{c}$. For a first order transition, one expects to find a bimodal $P(E)$, with peaks that sharpen to two separated $\delta$-functions as $L \rightarrow \infty$; these give the differing energies of the two coexisting phases. In Fig. 1 we plot the normalized $P(E)$ for sizes $L=120,160$, and 200, near $T_{c} \simeq 0.00229$. Analysis of the vortex structure function,

$$
S(\mathbf{k})=\frac{1}{f L^{2}} \sum_{i, j}\left\langle\mathrm{e}^{i \mathbf{k} \cdot\left(\mathbf{r}_{i}-\mathbf{r}_{j}\right)}\right\rangle,
$$

clearly shows that the lower (upper) energy peak corresponds to the pinned (floating) vortex lattice. For $L=120,160$, and 200 respectively, we have carried out $0.77,1.5$ and $4.7 \times 10^{8} \mathrm{MC}$ passes though the lattice to 
compute averages. These resulted in 2219, 1186 and 841 hops, respectively, between the two peaks. Having a large number of hops is important to achieve good equilibration of the relative weights of the two peaks (we have also done simulations for $L=80,100,140$, and 180). Qualitatively, we see that these peaks sharpen as $L$ increases, consistent with a 1st order transition. We now make this observation quantitative using several different criteria.

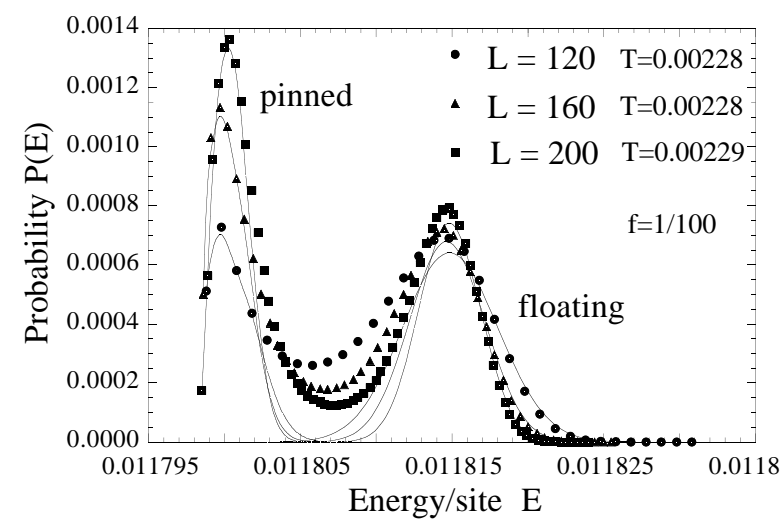

FIG. 1. Histograms of energy density $P(E)$ vs. $E$ for vortex density $f=1 / 100$, and system sizes $L=120,160$ and 200, at temperatures near the depinning transition $T_{c}$. The two peaks correspond to the coexisting pinned and floating solid phases. The peaks sharpen as $L$ increases, consistent with a 1st order transition. The solid lines are fits to the peaks, as described in the text.

For an infinite system, the bimodal $P(E)$ should exist only precisely at $T_{c}$, where the two phases coexist. For finite $L$, this coexistence region where $P(E)$ is noticeably bimodal persists over a finite temperature interval $\Delta T$. Since the relative weight of each peak is determined by the total free energy difference between the two phases, $\Delta F \sim L^{d}$, we expect the scaling $\Delta T \simeq 1 / \Delta F=1 / L^{d}=$ $1 / L^{2}$ in 2 D. Using standard methods [8] to extrapolate the histograms of Fig. 1 to nearby temperatures, we define the upper (lower) limit of the coexistence region, $T_{c \max (\min )}$, as the temperature at which the height of the lower (upper) peak has decreased to $1 \%$ the height of the upper (lower) peak. In Fig.2 we plot the results. We see that $T_{c \max }$ and $T_{c \text { min }}$ converge to a common value as $L$ increases, and that $\Delta T \sim 1 / L^{2}$ as expected for a 1 st order transition.
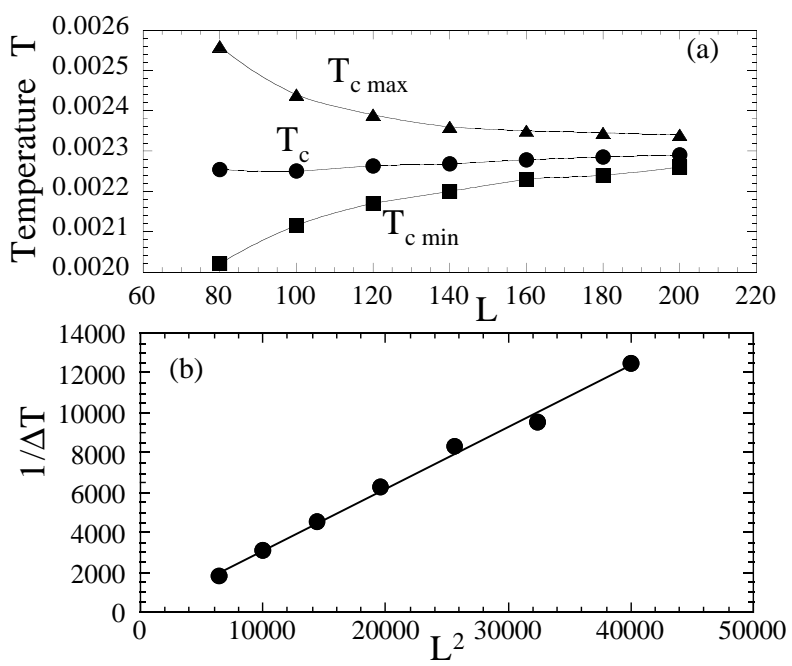

FIG. 2. (a) Plot of the upper and lower limits, $T_{c \max }$ and $T_{c \text { min }}$, of the coexistence region, and the finite size depinning temperature $T_{c}$, vs. system size $L$. (b) Inverse of the width of the coexistence region, $1 / \Delta T=1 /\left(T_{c \max }-T_{c \min }\right)$, vs. $L^{2}$. Vortex density is $f=1 / 100$. The straight line is a least squares fit, and the good agreement is consistent with a 1st order transition.

Next, we consider in detail how the two peaks sharpen as $L$ increases. For a 1 st order transition at $T_{c}$, the width $\sigma$ of each peak is determined by ordinary noncritical finite size fluctuations giving, $\sigma \sim 1 / L^{d / 2}=1 / L$ in 2 D. The total width of the bimodal $P(E), \sigma_{\text {tot }}$, approaches a constant (this is equivalent to the familiar observation that the specific heat $c \equiv L^{d} \sigma_{\text {tot }} / T \sim L^{d}$ at a 1 st order transition). To verify these scaling behaviors, we need to deconvolve the $P(E)$ of Fig. 1 into two separate peaks, and to determine $T_{c}(L)$ by the criteria that the two peaks subtend equal areas [9]. For large enough $L$, we expect each peak to have a Gaussian shape, and we have found this to give a good fit for the floating solid peak. The pinned solid peak however lies too close to the ground state energy $E_{0}$; even for our biggest size $L=200$, the states in this peak correspond to a few discrete excitations above the ground state. We therefore fit the pinned solid peak to the ad $h o c$ form, $\left(E-E_{0}\right) \exp \left[-\left(E-E_{1}\right)^{2} / 2 \sigma^{2}\right]$, where $E_{1}$ and $\sigma$ are fitting parameters. Using data from only the far side of each peak, we fit $P(E)$ to the sum of a Gaussian and the modified Gaussian above, to get the fitted curves shown in Fig. 1. From these fits we determine the probabilities that a state with a given value of $E$ belongs to the pinned phase, the floating phase, or neither (the later being the "transition" states, consisting of large domains of one phase in a background of the other, which give rise to the transitions between the two phases). We then go though the ensemble of states that enter into our averages, and probabilistically assign each state to the pinned phase, the floating phase, or neither. With this deconvolution, we then extrapolate to the $T_{c}(L)$ which gives equal weight to the two phases, and we can then 
compute the widths $\sigma_{\text {float }}, \sigma_{\text {pin }}$, and $\sigma_{\text {tot }}$ at this temperature. Our result for $T_{c}(L)$ is shown in Fig.2 $2 a$. Our results for the widths are shown in Fig. 3. We find $\sigma_{\text {tot }} \sim$ constant, and $\sigma_{\text {float }}, \sigma_{\text {pin }} \sim 1 / L$, as expected for a first order transition [10]. We can also use this decomposition to compute the entropy jump per vortex at the transition, $\Delta s=\left(\langle E\rangle_{\text {float }}-\langle E\rangle_{\text {pin }}\right) /\left(f T_{c}\right)$. Our results, shown in Fig. 4, give a $\Delta s(L)$ that saturates to a constant as $L$ increases, again as expected for a 1st order transition.

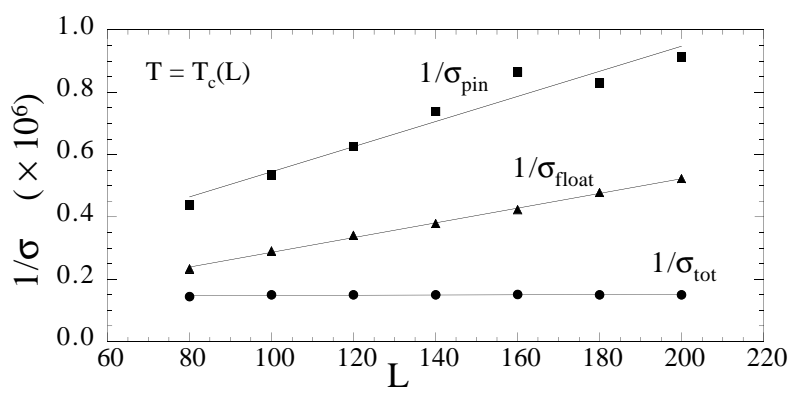

FIG. 3. Inverse of the total width $\sigma_{\text {tot }}$ of the energy histogram $P(E)$, and inverse of the widths, $\sigma_{\text {pin }}$ and $\sigma_{\text {float }}$, of the pinned and floating solid peaks, vs. system size $L$. Vortex density is $f=1 / 100$. The straight lines are least squares fits. The observed scaling $1 / \sigma_{\text {tot }} \sim$ constant, $1 / \sigma_{\text {pin }}, 1 / \sigma_{\text {float }} \sim L$ is consistent with a 1 st order transition.

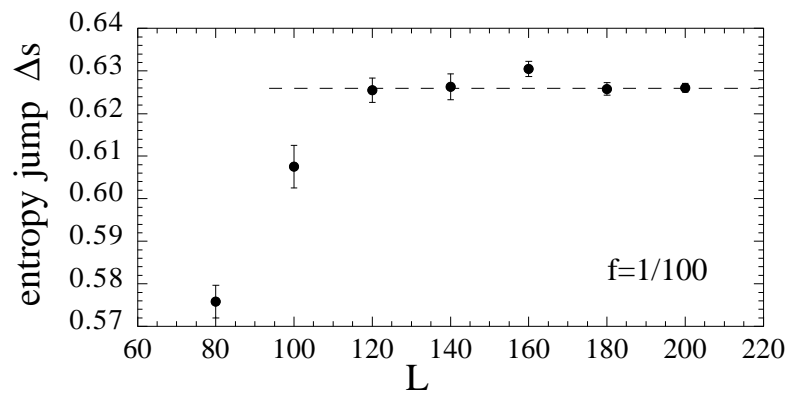

FIG. 4. Entropy jump per vortex, $\Delta s=\left(\langle E\rangle_{\text {float }}-\langle E\rangle_{\text {pin }}\right) /\left(f T_{c}\right)$, at the depinning transition vs. system size $L$. The vortex density is $f=1 / 100$. The dashed line is a guide to the eye only.

Next we consider the translational correlations. In the floating solid phase correlations are algebraic [2], $\left\langle\exp \left[i \mathbf{K} \cdot\left(\mathbf{r}_{i}-\mathbf{r}_{j}\right)\right] \sim\left|\mathbf{r}_{i}-\mathbf{r}_{j}\right|^{-\eta_{K}(T)}\right.$, with $\mathbf{K}$ a reciprocal lattice vector, and $\eta_{K} \equiv \eta|\mathbf{K}|^{2} /\left|\mathbf{K}_{1}\right|^{2}$, with $\mathbf{K}_{1}$ the smallest non-zero $\mathbf{K}$. Substituting this into the structure function, Eq.(2) results in,

$$
\frac{S(\mathbf{K})}{L^{2}} \sim L^{-\eta_{K}} \sim \mathrm{e}^{-(\eta \ln L)|\mathbf{K}|^{2} /\left|\mathbf{K}_{1}\right|^{2}} .
$$

According to the NH theory [2], $\eta$ should take a discontinuous jump from zero in the pinned solid to a universal finite value at $T_{c}$ in the floating solid. For an incompressible system such as ours, in which the bulk modulus $\lambda=\infty$, the predicted jump is,

$$
\eta\left(T_{c}^{+}\right)=4 f,
$$

where $f$ is the vortex density. From Eq.(3) we see that $S\left(\mathbf{K}_{1}\right) / L^{2}$ vs. $L$ plotted on a log-log scale should yield a straight line of negative slope $\eta(T)$. In Fig. Fa we show such plots for several values of $T$ near $T_{c}$, using $S\left(\mathbf{K}_{1}\right)$ averaged over all configurations encountered in the simulation. The straight lines are a least squares fit to the assumed algebraic form. We see that the fit is not very good, except perhaps at the highest temperatures. If, however, we average $S\left(\mathbf{K}_{1}\right)$ separately over only the states in the floating solid phase, and over only the states in the pinned solid phase, we see the expected behavior as shown in Fig. $5 \mathrm{~b}$. In the pinned phase, the curves saturate to a finite value as $L$ increases, reflecting the long range order of this phase. In the floating phase, the curves give good straight line fits, showing algebraically decaying correlations. From these fits, we extract the exponent $\eta$ of the floating phase, which we plot vs. $T$ in Fig. 6. We see that $\eta\left(T_{c}^{+}\right)$is close to, but noticeably above, the NH prediction of Eq.(1). Since the NH mapping onto a vector Coulomb gas inverts the temperature scale, this is consistent with a 1st order transition preempting the NH defect unbinding transition, with Eq.(4) serving as a lower bound on $\eta(T)$.
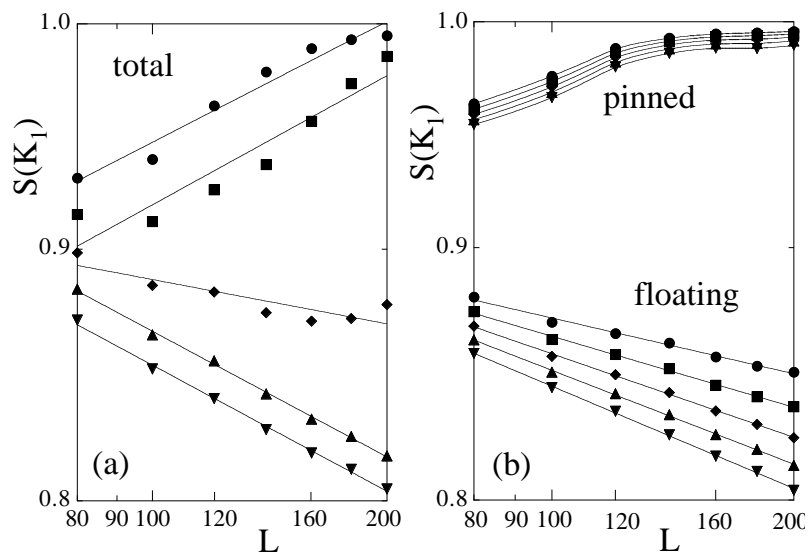

FIG. 5. Log-log plot of $S\left(\mathbf{K}_{1}\right)$ vs. $L$, for several values of temperature near $T_{c}$. (a) Shows $S\left(\mathbf{K}_{1}\right)$ averaged over all configurations; (b) shows $S\left(\mathbf{K}_{1}\right)$ averaged separately over only the pinned and only the floating configurations. From top to bottom the temperatures are, $T=0.00222,0.00226,0.00230$, 0.00234 and 0.00236 .

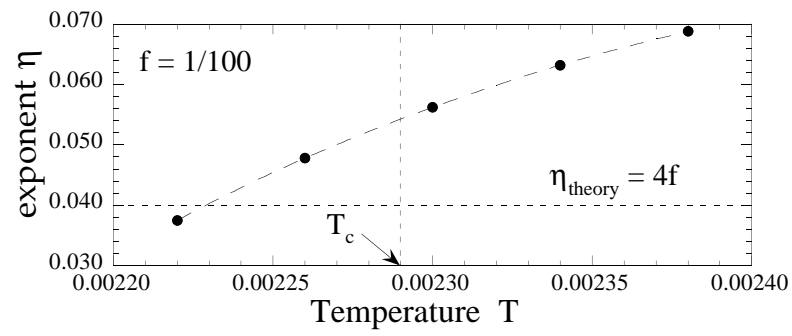


FIG. 6. Translational correlation exponent $\eta(T)$ of the floating lattice phase, as obtained from the fits in Fig. 5. The vortex density is $f=1 / 100 . \eta\left(T_{c}\right)$ remains above the theoretical lower limit $4 f$ of the Nelson Halperin theory.

The results above are all consistent with a 1st order transition rather than the 2 nd order $\mathrm{NH}$ prediction. However one can question whether this is an artifact of the particular density $f=1 / 100$ that we have used. Hattel and Wheatley 11] in particular have argued that, as $f$ decreases in the vortex system, the core energy of the defects that lead to depinning increases, and hence even if depinning is 1st order at some $f$, it must ultimately become 2nd order as $f$ decreases. To check this prediction we have carried out simulations for a variety of densities $f=49,64,100,196,400$. Rather than do finite size scaling for each case, we study only systems with a fixed total number of vortices $N_{v}$, corresponding to system sizes $L=\sqrt{N_{v} / f}$. We choose $N_{v}=144$ because our finite size studies of $f=1 / 100$ indicated this to be sufficiently large to be within the asymptotic large $L$ limit. Analyzing the data from these simulations in exactly the same manner as described above, we show in Fig. 7 our result for the entropy jump per vortex at depinning, $\Delta s$, as a function of density $f$. We see that $\Delta s$ is only weakly dependent on $f$, extrapolating to a finite $\Delta s \simeq 0.60$ as $f \rightarrow 0$.

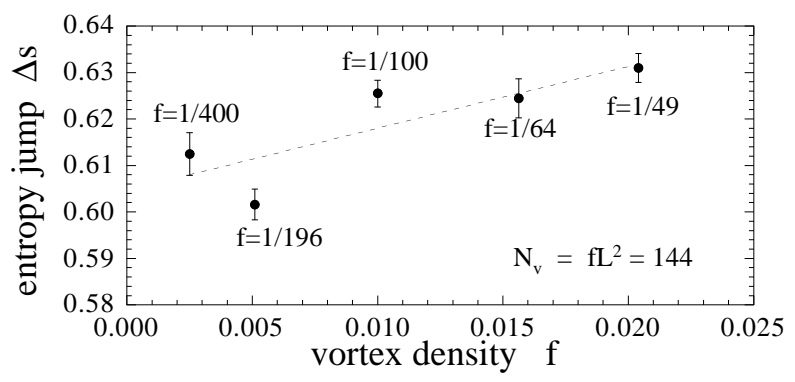

FIG. 7. Entropy jump per vortex, $\Delta s=\left(\langle E\rangle_{\text {float }}-\langle E\rangle_{\text {pin }}\right) /\left(f T_{c}\right)$, at the depinning transition vs. vortex density $f$, for systems with a fixed number of vortices $N_{v}=f L^{2}=144$. The dashed line is a least squares fit showing only a weak decrease in $\Delta s$ as $f$ decreases.

Finally, we consider the translational correlations for the different $f$. Since we have simulated only for fixed values of $f L^{2}$, we cannot extract $\eta$ by scaling with $L$ as in Fig. 5b. However we can estimate $\eta$ as follows. From Eq.(3) we expect a Gaussian envelope for the peaks $S(\mathbf{K})$ as a function of $|\mathbf{K}|$; the width gives a measure of $\eta$. This envelope is not purely Gaussian; a correction exists due to a $|\mathbf{K}|$ dependence of a prefactor in the $L^{-\eta_{K}}$ scaling of Eq.(3). Making a simple approximate correction for this effect, as done in Ref. [6], we plot our estimate for the correlation exponent, $\eta^{\prime}$, in Fig. 8. We show results using $S(\mathbf{K})$ averaged over all states, only the floating states, and only the pinned states, plotting the data in scaled units of $\eta^{\prime} / f$ vs. $T / T_{c}$. For $f=1 / 100$, our estimate $\eta_{\text {float }}^{\prime}$ is slightly larger than the more correct determination in Fig. 6. However the main point of Fig. 8 is to observe that the estimates $\eta^{\prime}$ all collapse to a common curve as the density $f$ decreases. There is no evidence for a continuing decrease in the value of $\eta\left(T_{c}^{+}\right)$as $f$ decreases, as might be the case if the 1st order transition was weakening and the NH prediction of Eq.(4) was approached more closely.

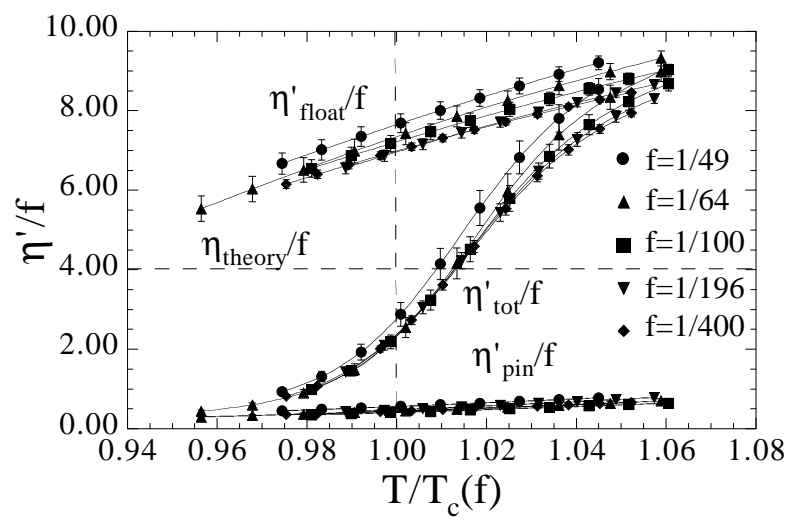

FIG. 8. Estimated exponent $\eta_{\text {tot }}^{\prime} / f, \eta_{\text {pin }}^{\prime} / f$, and $\eta_{\text {float }}^{\prime} / f$, as determined from $S(\mathbf{K})$ averaged over all states, the pinned solid states, and the floating solid states, respectively, vs. $T / T_{c}(f)$ for different vortex densities $f$. The data collapse to a common curve as $f$ decreases.

To conclude, a finite size scaling analysis for the density $f=1 / 100$ is strongly consistent with the depinning transition being 1 st order. We further find no evidence that this 1st order transition changes in any significant way if the density $f$ is decreased. We cannot rule out the possibility of different behavior for softer interactions than the logarithm considered here.

We would like to thank D. R. Nelson and M. Franz for very helpful conversations. This work was supported by the Engineering Research Program of the Office of Basic Energy Sciences at the Department of Energy, grant DEFG02-89ER14017.

[1] J. M. Kosterlitz and D. J. Thouless, J. Phys. C. 6, 1181 (1973).

[2] D. R. Nelson and B. I. Halperin, Phys. Rev. B 19, 2457 (1979).

[3] A. P. Young, Phys. Rev. B 19, 1855 (1979).

[4] For a review see, K. J. Strandburg, Rev. Mod. Phys. 60, 161 (1988).

[5] K. Bagchi, H. C. Anderson and W. Swope, Phys. Rev. E 53, 3794 (1996); A. Jaster, ibid. 59, 2594 (1999).

[6] M. Franz and S. Teitel, Phys. Rev. B 51, 6551 (1995).

[7] S. A. Hattel and J. M. Wheatley, Phys. Rev. B 51, 11951 (1995).

[8] A. M. Ferrenberg and R. H. Swendsen, Phys. Rev. Lett. 61, 2635 (1988); 63, 1195 (1989). 
[9] C. Borgs and R. Kotecký, J. Stat. Phys 61, 79 (1990).

[10] Although our results for $\sigma_{\text {pin }}$ depend directly on our choice of the ad hoc fitting form for the pinned peak, our results for $\sigma_{\text {tot }}$ and $\sigma_{\text {float }}$ depend only indirectly on it through the determination of $T_{c}(L)$; trying other fitting forms, we have found little change in the resulting $\sigma_{\text {tot }}$ and $\sigma_{\text {float }}$.

[11] S. Hattel and J. M. Wheatley, Phys. Rev. B 50, 16590 (1994). 